\title{
Magnetic Hopfions in the Faddeev-Skyrme-Maxwell model
}

\author{
A. Samoilenka ${ }^{1}$ and Ya. Shnir ${ }^{1,2}$ \\ ${ }^{1}$ Department of Theoretical Physics and Astrophysics, Belarusian State University, Minsk 220004, Belarus \\ ${ }^{2}$ BLTP, JINR, Dubna 141980, Moscow Region, Russia
}

(Received 1 May 2018; published 18 June 2018)

\begin{abstract}
We construct new solutions of the Faddeev-Skyrme-Maxwell model, which represent Hopf solitons coupled to magnetic fluxes. It turns out that coupling to the magnetic field allows for transmutations of the solitons; however, the results depend both on the type of the vacuum boundary condition and on the strength of the gauge coupling. It is shown that the structure of the magnetic fluxes of a gauged Hopfion is governed by the preimages of the points $\phi_{3}= \pm 1$.
\end{abstract}

DOI: 10.1103/PhysRevD.97.125014

\section{INTRODUCTION}

Topological solitons appear as classical solutions in various nonlinear models, and they have been intensively studied over the past decades. These regular localized field configurations with finite energy attracted a lot of attention; they emerge in a variety of physical, chemical, and biological systems.

Interesting examples of stable topological solitons exist in the family of Skyrme-type scalar theories, which can be considered as deformations of the nonlinear sigma model. It includes so-called baby Skyrmions in the $(2+1)$ dimensional $O(3)$ model [1,2], Skyrmions in the conventional $(3+1)$-dimensional Skyrme model [3] and its modifications [4,5], and the Hopfions in the FaddeevSkyrme model [6,7]. A unifying feature of all these models is that they have the same structure; the corresponding Lagrangians always include the usual $\sigma$-model term, the Skyrme term, which is quartic in derivatives of the field, and a potential term, which does not contain the derivatives. According to Derrick's theorem [8], the potential is optional in $3+1$ dimensions; however, it is obligatory to stabilize the soliton solutions of the planar baby-Skyrme model.

The solitons of the Faddeev-Skyrme model are somewhat special, because their topology is defined by the first Hopf map $S^{3} \mapsto S^{2}$ with the related homotopy group $\pi_{3}\left(S^{2}\right)=\mathbb{Z}$. It corresponds to the topological charge, which is the linking number of loops on the compactified domain space $S^{3}$.

Notably, all the models of the Skyrme family on a noncompact domain do not saturate the topological bound.

Published by the American Physical Society under the terms of the Creative Commons Attribution 4.0 International license. Further distribution of this work must maintain attribution to the author(s) and the published article's title, journal citation, and DOI. Funded by SCOAP.
In order to attain the bound which yields a relation between the static energy of the solitons and their topological charges $Q$, one has to modify the model, preserving its topological properties, for example, truncate the FaddeevSkyrme model [9] or, oppositely, extend the Skyrme model via coupling it to an infinite tower of vector mesons [10] or completely change the original theory to the form which supports self-dual equations $[4,5,11,12]$. Thus, the energy of interaction between the solitons is relatively large, and they may attract each other, forming various multisoliton configurations; see, e.g., [13,14].

Intuitively, the Hopfions can be constructed by considering baby Skyrmions restricted to the plane which is orthogonal to the direction of the position curve of the stringlike configuration [15]. The topological charge of such a soliton corresponds to the product of the winding number of the planar Skyrmions and the number of twists of the entire configuration in the extra spatial direction. Physically, solitons of that type can be considered as a vortex which is bending and twisting. The identification of the end points of the vortex yields the loop, which can transform itself into a knot to minimize its energy.

A peculiarity of the interaction potential, in the case of both Skyrmions and Hopfions, is that the asymptotic decay of the fields, which defines the character of interaction, strongly depends on the explicit form of the potential [4,5,16-20]. Furthermore, various symmetry-breaking potentials were considered to construct half-Skyrmions [21-25] or fractional Hopfions [26].

There is another possibility to make alterations to the structure of multisoliton solutions. In the Faddeev-Skyrme model, the vacuum boundary condition should be imposed in such a way that all the points on the boundary are identified. It yields the compactification of the domain space from $\mathbb{R}^{3}$ to $S^{3}$. Hence, the Hopfions are invariant with respect to the global $S O(2)$ symmetry of the vacuum. This allows us to construct the $U(1)$ gauged 
Faddeev-Skyrme-Maxwell theory by analogy with the extension of the gauged planar baby-Skyrme model [27-29]. Clearly, electromagnetic interaction will strongly affect the usual pattern of interaction in the system of Hopfions.

Unfortunately, the task of explicit construction of the solutions of the Faddeev-Skyrme-Maxwell theory has been hampered by numerous technical obstacles. Since there are no analytical solutions of the corresponding field equations, the minimizers of the corresponding energy functional can be obtained only numerically. However, it is known that the Hopfions of lowest degrees $Q=1,2$ are axially symmetric [30-32]; thus, in Ref. [33], the consideration was restricted to the case of the static axially symmetric gauged unlinked Hopfions $\mathcal{A}_{1,1}$ and $\mathcal{A}_{2,1}$. An assumption of axial symmetry simplifies the consideration significantly, since the problem then can be reduced to the numerical solution of the system of coupled ordinary differential equations. However, this symmetry is not a general property of general solutions of the Faddeev-Skyrme model supplemented by the Maxwell term; thus, this problem should be revisited.

In this paper, we investigate the structure of multisoliton solutions of the full coupled Faddeev-Skyrme-Maxwell system. Usually, there is an ambiguity in the choice of the topological boundary conditions on the scalar field; however, in the $U(1)$ gauged Faddeev-Skyrme model, it becomes dependent on the definition of the electromagnetic group. We consider two choices of the vacuum boundary conditions: $\vec{\phi}_{\infty}=(0,0,1)$ and $\vec{\phi}_{\infty}=(1,0,0)$. In both cases, we perform full 3D numerical computations to find the corresponding magnetic Hopf solitons in the sectors of degrees up to $Q=8$. We study numerically the dependence of masses of the Hopfions and the corresponding magnetic fluxes on the gauge coupling constant. We confirm that in the strong coupling limit the magnetic fluxes of the Hopfion become quantized in units of $2 \pi$.

We found that in a general case the magnetic fluxes of gauged Hopfions are defined by the preimages of the vectors $\vec{\phi}=(0,0, \pm 1)$, and there is an intrinsic interplay between the topology of the Hopf map and the structure of the magnetic field of the configuration.

The rest of the paper is structured as follows. In the next section, we briefly describe the Faddeev-SkyrmeMaxwell model. In Sec. III, for the sake of completeness, we review the rational map approximation used as input in our numerical simulations. Numerical results are presented in Sec. IV, where we describe various magnetic Hopfion solutions. For the sake of compactness, we restrict the analysis to the solitons with topological charges up to eight; as a particular example, we present a more detailed discussion of the evolution of the $Q=5$ Hopfions. Conclusions and remarks are formulated in the last section.

\section{II. $U(1)$ GAUGED FADDEEV-SKYRME MODEL}

We consider the Faddeev-Skyrme theory coupled to the Abelian gauge field in $(3+1)$ dimensions. The model is defined by the rescaled Lagrangian

$$
\mathcal{L}=\int d^{3} x\left[-\frac{1}{4 g^{2}} F_{\mu \nu}^{2}+D_{\mu} \vec{\phi} \cdot D^{\mu} \vec{\phi}-\frac{1}{2}\left(D_{\mu} \vec{\phi} \times D_{\nu} \vec{\phi}\right)^{2}\right],
$$

where the real scalar triplet $\vec{\phi}=\left(\phi_{1}, \phi_{2}, \phi_{3}\right)$ is constrained to the surface of a unit sphere, $|\vec{\phi} \cdot \vec{\phi}|=1$, so the target space is the sphere $S^{2}$. Since the potential term is optional, we do not consider it. However, the global $S O(3)$ symmetry will be broken as we impose the topological vacuum boundary conditions, like $\vec{\phi}_{\infty}=(0,0,1)$, which yield a one-point compactification of the domain space $\mathbb{R}^{3}$ to $S^{3}$. Note that this common choice is not unique; below, we will also consider another case, $\vec{\phi}_{\infty}=(1,0,0)$.

Thus, the field of the Hopfion is a map $\vec{\phi}: \mathbb{R}^{3} \rightarrow S^{2}$ which belongs to an equivalence class characterized by the homotopy group $\pi_{3}\left(S^{2}\right)=\mathbb{Z}$. Explicitly, the Hopf invariant is defined nonlocally as

$$
Q=\frac{1}{16 \pi^{2}} \int_{\mathbb{R}^{3}} \varepsilon_{i j k} \mathcal{F}_{i j} \mathcal{A}_{k},
$$

where $\mathcal{F}_{i j}=\vec{\phi} \cdot\left(\partial_{i} \vec{\phi} \times \partial_{j} \vec{\phi}\right)$ and one-form $\mathcal{A}=\mathcal{A}_{k} d x^{k}$ is defined via $\mathcal{F}=d \mathcal{A}$; i.e., the two-form $\mathcal{F}$ is closed, $d \mathcal{F}=0$.

The model (1) includes also the usual Maxwell term with the field strength tensor $F_{\mu \nu}=\partial_{\mu} A_{\nu}-\partial_{\nu} A_{\mu}$. Note that under a spatial rescaling $\mathbf{x} \rightarrow \lambda \mathbf{x}$, this term in the action scales as $\lambda^{-1}$; i.e., it has the same scaling properties as the quartic in the derivative Skyrme term. The flat metric is $g_{\mu \nu}=\operatorname{diag}(1,-1,-1,-1)$, and the coupling of the scalar triplet to the gauge field is given by the covariant derivative $[27,28,33,34]$

$$
D_{\mu} \vec{\phi}=\partial_{\mu} \vec{\phi}+A_{\mu} \vec{\phi} \times \vec{n},
$$

where the unit vector $\vec{n}=(0,0,1)$ defines the direction of the electromagnetic subgroup. Explicitly,

$$
D_{\mu} \phi_{\perp}=\partial_{\mu} \phi_{\perp}-i A_{\mu} \phi_{\perp}, \quad D_{\mu} \phi_{3}=\partial_{\mu} \phi_{3},
$$

where $\phi_{\perp}=\phi_{1}+i \phi_{2}$ are planar components of the scalar field. Thus, the third component $\phi_{3}$ remains decoupled from the gauge potential. However, since the scalar triplet is restricted to the surface of the unit sphere, coupling of the planar components $\vec{\phi}_{\perp}$ to the gauge sector still affects the component $\phi_{3}$ indirectly. 
The Abelian gauge transformations act on the fields as

$$
\phi_{\perp} \rightarrow e^{i \alpha} \phi_{\perp}, \quad A_{\mu} \rightarrow A_{\mu}+\partial_{\mu} \alpha,
$$

and, thus, we can make use of this symmetry to set $A_{0}=0$. Further restricting our analysis to static configurations, we consider purely magnetic field $\vec{B}=\left(-\partial_{3} A_{2}, \partial_{3} A_{1}, \partial_{1} A_{2}-\partial_{2} A_{1}\right)$.

The static energy functional of the model (1) is

$$
E=\int d^{3} x\left[\frac{1}{2 g^{2}} \vec{B}^{2}+D_{i} \vec{\phi} \cdot D_{i} \vec{\phi}+\frac{1}{2}\left(D_{i} \vec{\phi} \times D_{j} \vec{\phi}\right)^{2}\right] .
$$

Here we are using normalized units of energy, rescaling it as $E \rightarrow E /\left(32 \pi^{2} \sqrt{2}\right)$. The Hopfions correspond to the stationary points of this functional. Note that the condition of finiteness of energy implies that $D_{i} \phi_{\perp}=\partial_{i} \phi_{\perp}-$ $i A_{i} \phi_{\perp} \underset{r \rightarrow \infty}{\rightarrow} 0$ as $r \rightarrow \infty$. In other words, on the spatial asymptotic, the field of the gauged Hopfion must lie in an orbit of the gauge group; it is not necessarily a constant there.

The complete set of the field equations, which follows from the variation of the action of the model (1), is

$$
D_{\mu} \vec{J}^{\mu}=0 ; \quad \partial_{\mu} F^{\mu \nu}-2 g^{2} \vec{n} \cdot \vec{J}^{\nu}=0 .
$$

Here the scalar current is

$$
\vec{J}^{\mu}=\vec{\phi} \times D^{\mu} \vec{\phi}-D_{\nu} \vec{\phi}\left(\vec{\phi} \cdot D^{\mu} \vec{\phi} \times D^{\nu} \vec{\phi}\right),
$$

and a source in the corresponding Abelian Maxwell equations is $j_{\mu}=\vec{n} \cdot \vec{J}_{\mu}$. This system is similar to the corresponding equations of the planar Skyrme-Maxwell theory $[27,29,34]$; however, the topological properties of the fields are different.

Unlike other solitons, the location of the Hopfions does not correspond to the maximum of the topological charge density; the Hopfions are extended stringlike configurations in three-dimensional space. The maxima of the energy density distribution can be identified as the curve of positions of the preimage of the point $\vec{\phi}_{0}=$ $(0,0,-1)$, which is antipodal to the vacuum [32]. This curve is usually referred to as the position curve [32]. In the gauged Faddeev-Skyrme model, this curve has also another meaning.

Note that we can make use of the trigonometrical parametrization of the scalar field

$$
\vec{\phi}=(\sin \psi \cos \sigma, \sin \psi \sin \sigma, \cos \psi),
$$

where two functions $\psi(x, y, z)$ and $\sigma(x, y, z)$ satisfy the boundary conditions on the Hopfion configuration in a given topological sector. Although this parametrization is not the most convenient from the point of view of numerical simulations [35], it automatically takes into account restriction of the scalar field to $S^{2}$. This, in particular, allows for a more transparent understanding of many peculiarities of the gauged Faddeev-Skyrme model.

In a simple case of the axially symmetric gauged Hopfions $\mathcal{A}_{1,1}$ and $\mathcal{A}_{2,1}$ [30], the function $\sigma$ can be explicitly written in spherical coordinates $(r, \theta, \varphi)$ as $\sigma=n \varphi-m G(r, \theta)$, where two winding numbers $n, m \in$ $\mathbb{Z}$ correspond to the planar winding and the twisting of the configuration, respectively. The phase function of the axially symmetric configuration $G(r, \theta)$ increases by $2 \pi$ after one revolution around the core; thus, the Hopf number of the soliton is just a product of two windings, $Q=m n$, and the axially symmetric configuration of the type $\mathcal{A}_{m, n}$ can be thought of as composed from a planar baby Skyrmion of charge $n$ twisted $m$ times along the circle [30].

In a general case, by analogy with the similar situation in the gauged planar Skyrme model [36], the Abelian current can be written as

$$
j_{i}=\left(\partial_{i} \sigma-A_{i}\right)\left[1-\phi_{3}^{2}+\partial_{j} \phi_{3}^{2}\right]-\partial_{i} \phi_{3} \partial_{j} \phi_{3}\left(\partial_{j} \sigma-A_{j}\right) .
$$

We can assume that the gauge potential $A_{i}$ slowly varies in space. Then, from the second equation in (7), we can see that in the limit of infinitely large gauge coupling $g \rightarrow \infty$ this equation is satisfied only if the Abelian current (10) becomes zero. Evidently, if $\phi_{3} \neq \pm 1$, the current is vanishing when $A_{i}=\partial_{i} \sigma$; i.e., the magnetic potential becomes a pure gauge everywhere in 3D space apart from the curves $\mathcal{C}_{ \pm}=\phi^{-1}(0,0, \pm 1)$.

Considering the magnetic flux through the area, transverse to the direction of the $\phi_{3}$, we can see that

$$
\Phi=\int B d^{2} x=\oint_{\Gamma} \vec{A} \cdot \overrightarrow{d l}=\oint_{\Gamma} \nabla \sigma \cdot \overrightarrow{d l}=2 \pi n,
$$

where $\Gamma$ is a closed contour encircling the points, where $\vec{\phi}=(0,0, \pm 1)$.

The consideration above explains the effective quantization of the magnetic fluxes of the gauged axially symmetric Hopfions of degrees $Q=1,2$ in the strong coupling limit [33]. It was observed that the configuration is associated with two magnetic fluxes, one of which represents a circular vortex, and the second one is orthogonal to the position curve [37]. In the strong coupling limit, the former flux is quantized in units of the winding number $n$, while the latter flux is quantized in units of $m$.

Indeed, the position curve of the Hopfion is defined as the preimage of the point $\phi=(0,0,-1)$ on the target space. On the other hand, for the axially symmetric Hopfions, the component $\phi_{3}$ is approaching the vacuum on the symmetry axis, so $\phi=(0,0,1)$ as $r=0$. Therefore, there are two associated magnetic fluxes, both becoming quantized in the strong coupling limit. More generally, the 
curves of $\phi_{3}= \pm 1$ define the structure of the magnetic fluxes of a gauged magnetic Hopfion.

\section{INITIAL APPROXIMATION}

The task of finding of multisoliton solutions of the Faddeev-Skyrme model in a given sector of degree $Q$ is very complicated; it can be performed only numerically. Moreover, it involves a rather sophisticated numerical technique; see, e.g., [32].

As usual, the energy minimization scheme needs an appropriate initial configuration in a given sector. The most effective approach here is related with the rational map approximation, suggested by Sutcliffe [32]. One can consider two complex variables which parameterize the sphere $S^{3}[32]$ :

$$
\left(Z_{1}, Z_{0}\right)=\left(\sin f(r) \sin \theta e^{i \varphi} ; \cos f(r)+i \sin f(r) \cos \theta\right),
$$

where $f(r)$ is a monotonically decreasing function with the boundary values $f(0)=\pi$ and $f(\infty)=0$. The coordinates $Z_{1}, Z_{0}$ are restricted to the unit sphere $S^{3}$, i.e., $\left|Z_{1}\right|^{2}+\left|Z_{2}\right|^{2}=1$. This allows us to construct a map $\mathbb{R}^{3} \mapsto S^{3} \in \mathbb{C}^{2}$.

The components of the scalar field $\vec{\phi}$, which are coordinates on the target space $S^{2}$, are given by the rational map $W: S^{3} \in \mathbb{C}^{2} \mapsto C P^{1}$ :

$$
W\left(Z_{1}, Z_{0}\right)=\frac{\phi_{1}+i \phi_{2}}{1+\phi_{3}}=\frac{P\left(Z_{1}, Z_{0}\right)}{Q\left(Z_{1}, Z_{0}\right)},
$$

where the polynomials $P\left(Z_{1}, Z_{0}\right)$ and $Q\left(Z_{1}, Z_{0}\right)$ have no common roots on the two-sphere $S^{2}$. The rational map ansatz (13) produces a curve in $\mathbb{R}^{3}$; therefore, the first Hopf map $\vec{\phi}: \mathbb{R}^{3} \mapsto S^{2}$ is equivalent to the rational map $W: S^{3} \mapsto C P^{1}$.

There are three different types of input configurations. The axially symmetric Hopfions $\mathcal{A}_{m n}$ are produced by the rational map [32]

$$
W\left(Z_{1}, Z_{0}\right)=\frac{Z_{1}^{n}}{Z_{0}^{m}}
$$

This Hopfion has a single position curve $\mathcal{C}_{-}=\phi^{-1}(0,0,-1)$.

More generally, we can consider initial configurations, which are given by maps of the form

$$
W\left(Z_{1}, Z_{0}\right)=\frac{Z_{1}^{\alpha} Z_{0}^{\beta}}{Z_{1}^{a}+Z_{0}^{b}},
$$

where $\alpha$ is a positive integer and $\beta$ is a non-negative integer. These maps have Hopf degree $Q=\alpha b+\beta a$, and the corresponding configuration is a torus knot $\mathcal{K}_{a, b}$. In a particular case when $a$ and $b$ are not coprime integers, the rational map (15) is degenerated, producing a link with two or more interlinked and disconnected position curves. Configurations of that type are labeled as $\mathcal{L}_{a, b}^{n, m}$, where the subscripts label the Hopf indexes of the unknots and the superscript above each subscript counts the secondary linking number, which appears due to interlinking with the other components.

Note that the location of the soliton can be identified as a collection of curves, which follow the preimages of two distinct points, for example, $\mathcal{C}_{-}=\vec{\phi}^{-1}(0,0,-1)$ and $\mathcal{C}_{1}=\vec{\phi}^{-1}(1,0,0)$. Since these loops are linked $Q$ times, the definition of the linking number can be related with the positions of the preimages of these points: $Q=\operatorname{link}\left(\mathcal{C}_{-}, \mathcal{C}_{1}\right)$. Other choices of the preimages are also possible [26].

The input for the magnetic potential $A_{i}$ at a finite value of the gauge coupling $g$ can be taken as a generalization of the limiting form of the pure gauge condition above:

$$
A_{i}=\partial_{i} \sigma\left(\phi_{\perp}\right) A\left(\phi_{3}\right) \underset{g \rightarrow \infty}{\rightarrow} \partial_{i} \sigma\left(\phi_{\perp}\right),
$$

where the function $\sigma\left(\phi_{\perp}\right)$ appeared in the trigonometric parametrization (9) of the scalar fields. The smooth function $A\left(\phi_{3}\right)$ must satisfy the restrictions $A( \pm 1)=0$ and $A(0) \simeq 1$, and it agrees with the parametrization used previously to construct axially symmetric solutions of the gauged Faddeev-Skyrme model [33]. Thus, we can take $A\left(\phi_{3}\right)=1-\phi_{3}^{2}$ as an appropriate choice.

\section{NUMERICAL RESULTS}

For our numerical computations, we used the algorithm of minimization of the energy functional (6) described in Refs. [28,38]. The fields are discretized on the grid with $100^{3}$ or $150^{3}$ points with grid spacing $\Delta x=0.1$. The initial configurations were produced via the rational map approximation as above. As a consistency check, we verify that our algorithm correctly reproduces the known results for the Hopfion configurations of the usual decoupled Faddeev-Skyrme model at $g=0$ and for the gauged axially symmetric configurations $\mathcal{A}_{11}$ and $\mathcal{A}_{21}$ previously discussed in Ref. [33].

The solutions of that type, $\mathcal{A}_{11}$ and $\mathcal{A}_{21}$, are global minima in the sectors of degrees $Q=1,2$, respectively. They represent axially symmetric unknots with the position curve $\mathcal{C}_{-}=\phi^{-1}(0,0,-1)$ forming a single loop. For the configuration $\mathcal{A}_{21}$, the corresponding linking curve, associated with the preimage of the point $\mathcal{C}_{1}=\phi^{-1}(1,0,0)$, has two twists around the position curve, as shown in Fig. 1.

Note that, with the usual choice of the vacuum boundary conditions $\vec{\phi}_{\infty}=(0,0,1)$, the vector $\vec{n}$, which appears in the definition of the covariant derivative (3), is parallel to $\vec{\phi}_{\infty}$. Below, we will also consider another situation, when 


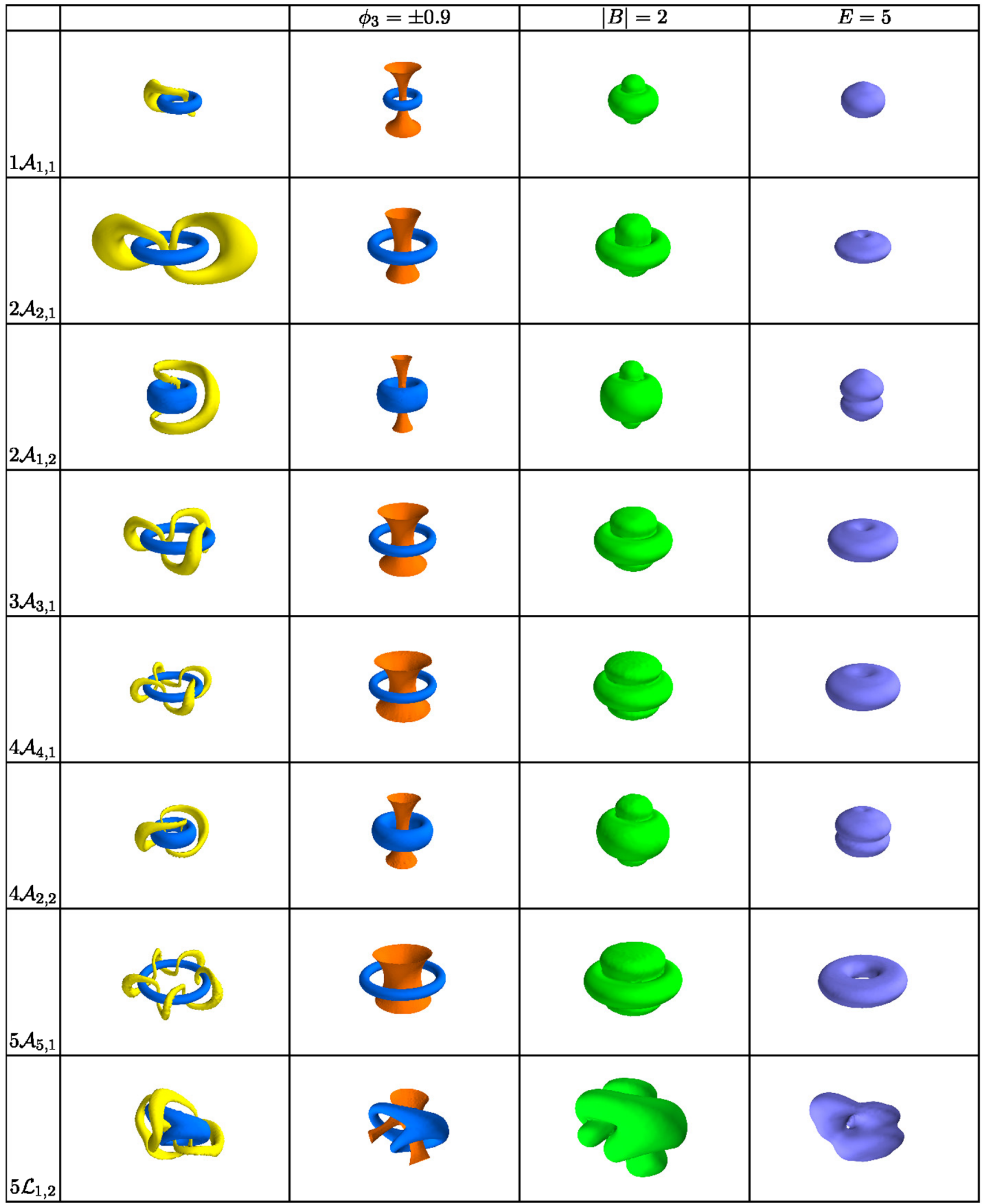

Fig. 1. (Continued). 


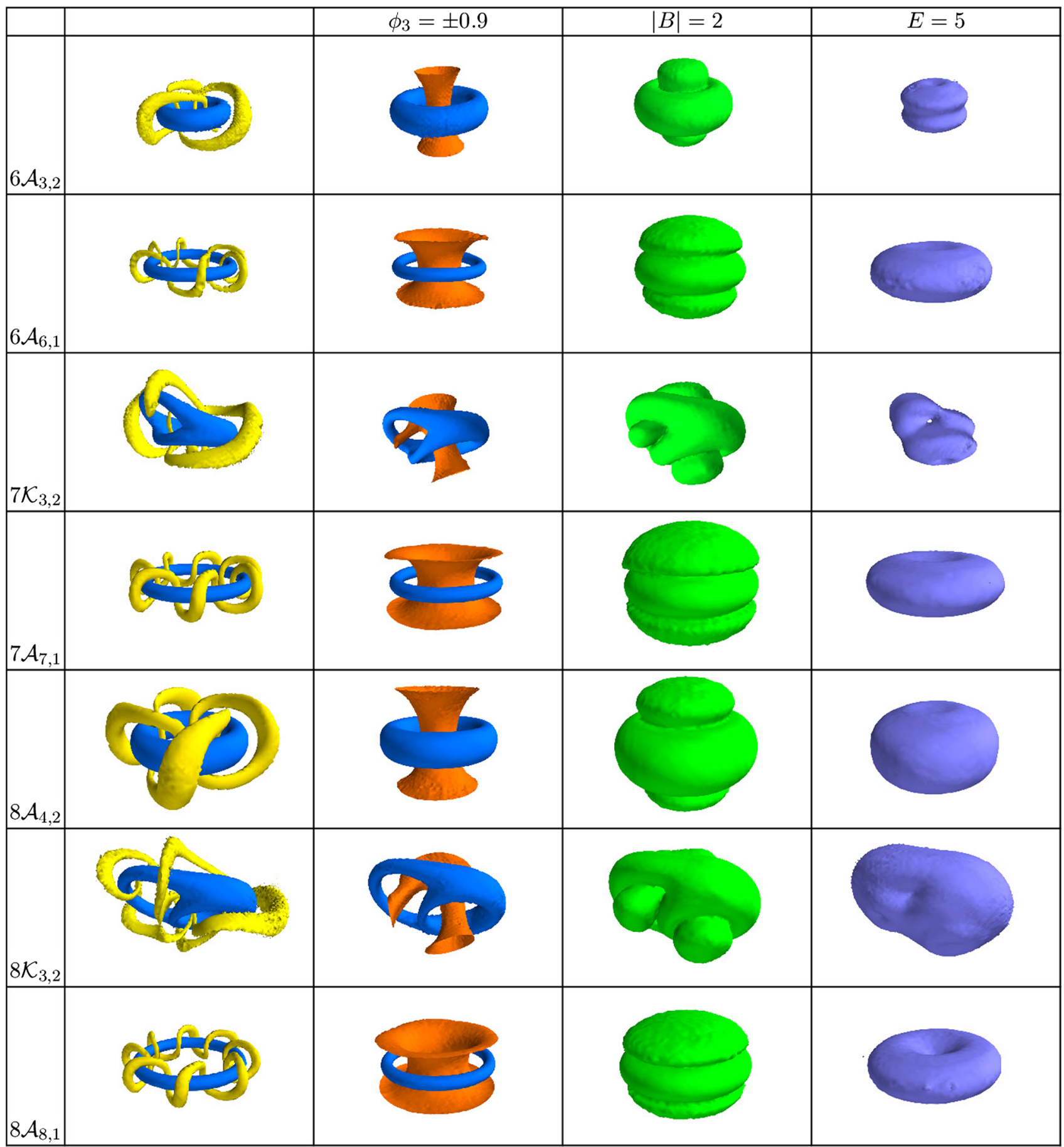

FIG. 1. Isosurfaces of the field components $\phi_{1}=-0.9$ and $\phi_{3}=-0.9$ (first column), the field components $\phi_{3}= \pm 0.9$ (second column), $|B|=2$ isosurfaces of the magnetic field (third column), and $E=5$ isosurfaces of the energy density (fourth column) for $Q=1-8$ gauged Hopfions in the model (1) with $g=1$ and $\vec{\phi}_{\infty}=(0,0,1)$.

on the spatial boundary $\vec{\phi}_{\infty}=(1,0,0)$ and $\vec{n}$ is transverse to $\vec{\phi}_{\infty}$.

As the gauge coupling gradually increases from $g=0$, the energy of the configuration decreases, since the magnetic flux is formed and the core of the Hopfions shrinks. The magnetic energy is initially increasing; however, its contribution starts to decrease as $g$ becomes larger than $g=1$ [33]. The structure of the magnetic field follows the pattern above, in the weak coupling regime there is a toroidal magnetic field, which encircles the position curve of the Hopfion. As the gauge coupling constant increases, the curves of the preimages of the points $\mathcal{C}_{ \pm}=\vec{\phi}^{-1}(0,0, \pm 1)$ pave the way for magnetic flux tubes. 


\begin{tabular}{|c|c|c|c|}
\hline Configuration & $\phi_{3}= \pm 0.9$ & $|B|=2$ & $E=5$ \\
\hline $1 \mathcal{A}_{1,1}$ & & & \\
\hline $2 \mathcal{A}_{2,1}$ & & & \\
\hline $2 \mathcal{A}_{1,2}$ & & & \\
\hline $3 \mathcal{A}_{3,1}$ & & & \\
\hline $4 \mathcal{A}_{2,2}$ & & & \\
\hline $4 \mathcal{A}_{4,1}$ & & & \\
\hline $5 \mathcal{L}_{1,2}$ & & & \\
\hline $5 \mathcal{A}_{5,1}$ & & & \\
\hline
\end{tabular}

Fig. 2. (Continued). 


\begin{tabular}{|l|l|l|l|}
\hline Configuration & $\phi_{3}= \pm 0.9$ & $|B|=2$ & $E=5$ \\
\hline $6 \mathcal{A}_{6,1}$ & & & \\
\hline $8 \mathcal{L}_{2,2}$ & & & \\
\hline $7 \mathcal{K}_{3,2}$ & & & \\
\hline $8 \mathcal{A}_{4,2}$ & & \\
\hline
\end{tabular}

FIG. 2. Isosurfaces of the field components $\phi_{1}= \pm 0.9$ (left column), $|B|=2$ isosurfaces of the magnetic field (middle column), and $E=5$ isosurfaces of the energy density (right column) for $Q=1-8$ gauged Hopfions in the model (1) with $g=1$ and $\vec{\phi}_{\infty}=(1,0,0)$.

Indeed, we can clearly identify two fluxes along these curves; the first flux is directed along the symmetry axis of the configuration, and the second circular magnetic flux is orthogonal to the $x-z$ plane (see Fig. 1). Both fluxes become quantized in units of $2 \pi$ in the strong coupling limit [33].

Further increase of the gauge coupling leads to shrinkage of the Hopfion, and the magnetic field effectively squeezes the configuration. This effect is opposite to the isorotations of the Hopfions, which also affect the structure of the solutions $[39,40]$.

Note that there is another axially symmetric Hopfion configuration in the sector of degree two, $2 \mathcal{A}_{12}$ $[30,32,41,42]$. It can be thought of as two $Q=1$
Hopfions stacked one above the other. In the limit $g=0$, this solution is a saddle point configuration, which has a higher energy than the $\mathcal{A}_{21}$ Hopfion. As $g$ increases, it still remains as a saddle point (see Fig. 3).

Interestingly, there is a certain similarity between the structure of the magnetic field of $Q=1,2$ axially symmetric Hopfions and toroidal magnetic fields which are well known in solar and plasma physics; see, e.g., [43]. In the latter case, the magnetic field appears as a solution of the so-called force-free equation for a plasma current $\vec{j} \times \vec{B}=0$.

In order to see the difference between the cases of the magnetic field of the gauged Hopfions and the magnetic 


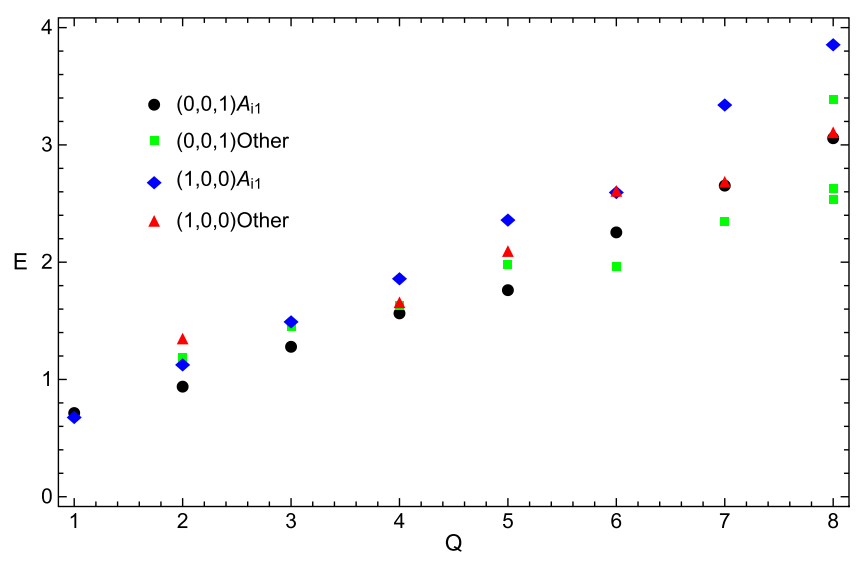

FIG. 3. Energy of $Q=1-8$ Hopfions with $g=1$ and $\vec{\phi}_{\infty}=$ $(0,0,1)$ and $\vec{\phi}_{\infty}=(1,0,0)$.

field in a plasma device, such as a stellerator or tokamak, let us assume that the scalar current $\vec{j}$ is a plasma current. However, the field-free equation leads to $\vec{B}=\alpha \vec{j}$; thus, for a constant $\alpha$ we obtain the Helmholtz equation:

$$
\Delta \vec{B}+\alpha^{2} \vec{B}=0
$$

On the other hand, the magnetic field of the Hopfions is generated by the scalar current (8), which can be written as

$$
\vec{j}=\vec{K}\left(1-\phi_{3}^{2}\right)-\vec{\nabla} \phi_{3} \times\left(\vec{\nabla} \phi_{3} \times \vec{K}\right),
$$

where $\vec{K}=\vec{\nabla} \sigma-\vec{A}$. Thus, the corresponding Maxwell equation becomes

$$
\vec{\nabla} \times(\vec{\nabla} \times \vec{K})-2 g^{2} \vec{\nabla} \phi_{3} \times\left(\vec{\nabla} \phi_{3} \times \vec{K}\right)+2 g^{2} \vec{K}\left(1-\phi_{3}^{2}\right)=0 .
$$

Since in the strong coupling regime the magnetic flux tubes follow the directions of the curves of preimages of the vectors $\vec{\phi}=(0,0, \pm 1)$, we can assume that outside of these curves $\phi_{3} \simeq 0$, and thus

$$
\vec{\nabla} \times(\vec{\nabla} \times \vec{K})+2 g^{2} \vec{K} \simeq 0 .
$$

Since $\vec{B}=\vec{\nabla} \times \vec{A}=-\vec{\nabla} \times \vec{K}$, we can see that the counterpart of the force-free equation for the magnetic field of the Hopfions can be written as

$$
\Delta \vec{B}-2 g^{2} \vec{B} \simeq 0 .
$$

Notably, this is a London-type equation with the penetration depth parameter $\frac{1}{\sqrt{2} g}$, and the mass term here has a sign opposite to the one in the force-free equation (17). Thus, in the strong coupling limit, the magnetic field of a Hopfion exhibit a sort of Meissner effect.

\section{A. Higher-charge gauged Hopfions at $\vec{\phi}_{\infty}=(0,0,1)$}

Peculiar feature of the Hopfions of higher degrees is that in the standard Faddeev-Skyrme model they usually do not possess any symmetry [32], and the corresponding collection of position curves is not planar. For example, for the charge three Hopfion, the energy minimization transforms the corresponding axially symmetric initial configuration $3 \mathcal{A}_{31}$ into the pretzel-like Hopfion $3 \tilde{\mathcal{A}}_{31}$.

Let us now consider the $Q=3$ Hopfion solution in the Faddeev-Skyrme-Maxwell model (1) with the usual boundary condition $\vec{\phi}_{\infty}=(0,0,1)$. As the gauge coupling constant gradually increases from zero, the position curve, initially bending toward the third direction, smoothly becomes a planar loop; see Fig. 1. The axially symmetric gauged Hopfion $3 \mathcal{A}_{31}$ becomes the global minimum in this sector at $g \sim 0.3$; as the gauge coupling increases further, the deformed Hopfion $3 \tilde{\mathcal{A}}_{31}$ does not exist as a local minimum.

Note that, similar to the case of the $Q=1,2$ Hopfions, the total energy of the configurations of higher degrees decreases as the gauge coupling $g$ increases, and this observation holds in a general case; see Fig. 4.

For Hopf degree $Q=4$, there are possibilities to construct initial configurations of types $4 \mathcal{A}_{22}, 4 \mathcal{A}_{41}$, and $4 \mathcal{L}_{11}^{11}$. In the usual Faddeev-Skyrme model without the magnetic field, the axially symmetric Hopfion $\mathcal{A}_{22}$, which may be thought of as two adjacent $2 \mathcal{A}_{21}$ solitons in the maximally attractive channel of interaction, represents the global minimum [32]. Numerical relaxation of the initial $4 \mathcal{A}_{41}$ configuration yields a buckled Hopfion $4 \tilde{\mathcal{A}}_{41}$; however, in the limit $g=0$, its energy is about $2 \%$ above the global minimum. The situation changes as the gauge coupling increases; the interaction with the magnetic field tends to straighten out the position curve, and thus the axially symmetric Hopfion $4 \mathcal{A}_{41}$ has a lower energy than $4 \mathcal{A}_{22}$.

In the usual Faddeev-Skyrme model at $g=0$, in the sector of degree four, the link $4 \mathcal{L}_{1,1}^{1,1}$ does not exist as a local minimum. However, this type of solution $5 \mathcal{L}_{2,1}^{1,1}$ is a minimizer for $Q=5$ Hopfions. We observe that an increase of the gauge coupling transforms it into configuration of a different type. As $g \sim 1$, the magnetic attraction between the fluxes, associated with the collection of loops $\mathcal{C}_{-}=\phi^{-1}(0,0,-1)$, deforms the position curve; it corresponds to two adjacent loops which are not linked to each other (see Fig. 1). Furthermore, such a configuration is not a global minimum in this sector; as $g \gtrsim 0.2$, the axially symmetric configuration $\mathcal{A}_{51}$ has a lower energy (see Fig. 4).

Note that the magnetic field of all axially symmetric gauged Hopfions $\mathcal{A}_{n 1}$ represents two magnetic fluxes: one flux encircles the position curve of the Hopfion, and the second one is directed along the symmetry axis; this pattern is illustrated in Fig. 1. As we discussed above, in the strong 

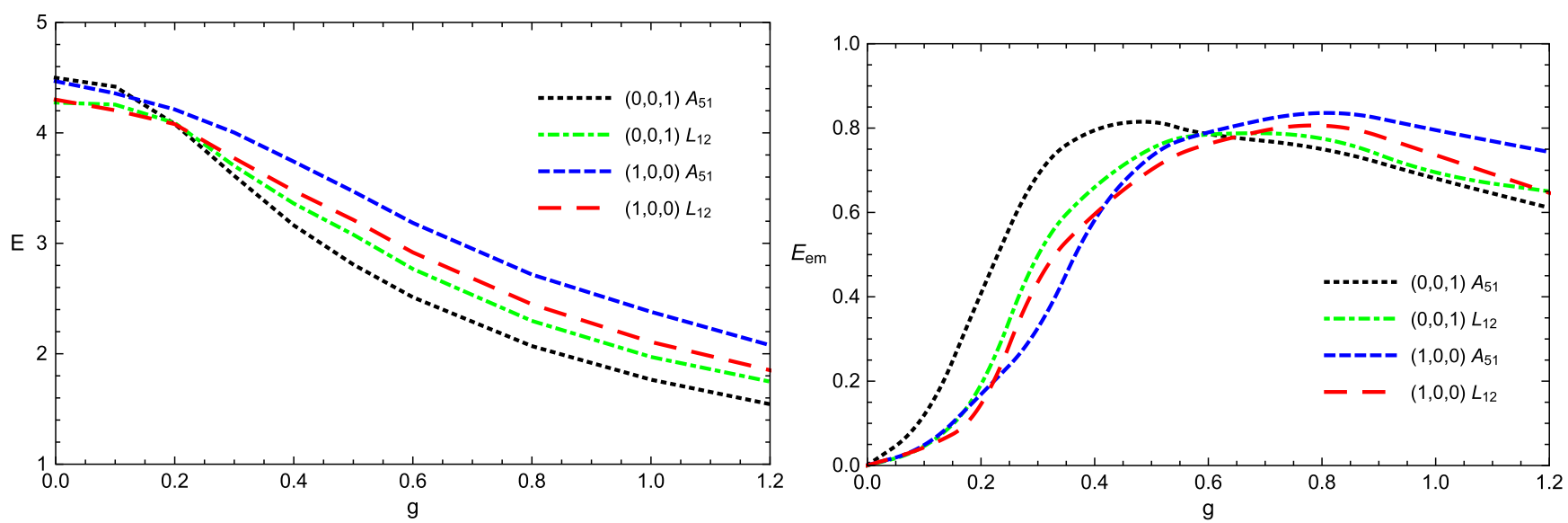

FIG. 4. The normalized energy $E$ of the $5 \mathcal{A}_{5,1}$ and $5 \mathcal{L}_{1,2}^{1,1}$ gauged Hopfions (left plot) and the corresponding magnetic energy (right plot) as a function of the coupling constant $g$ in the Faddeev-Skyrme-Maxwell model (1) for the vacua $\vec{\phi}_{\infty}=(0,0,1)$ and $\vec{\phi}_{\infty}=(1,0,0)$.

coupling limit, both fluxes are quantized in units of $2 \pi$ and $2 \pi n$, respectively.

Considering the structure of the magnetic field of an $\tilde{\mathcal{A}}_{n 1}$ Hopfion with axial symmetry weakly broken, we observe that one of the magnetic fluxes follows the position curve $\mathcal{C}_{-}=\phi^{-1}(0,0,-1)$. Another flux is associated with the direction of the vector $\vec{n}=(0,0,1)$ in (3). This is also the case of the $Q=5$ Hopfion; as seen in the ninth row in Fig. 1, the magnetic fluxes follow the loops, which are preimages of $\mathcal{C}_{-}=\vec{\phi}^{-1}(0,0,-1)$ and $\mathcal{C}_{+}=\vec{\phi}^{-1}(0,0,1)$.

The axially symmetric configurations $\mathcal{A}_{n 1}$ represent global minima in the strong coupling regime up to $Q \leq 5$. When $Q=6$, there are two different links $6 \mathcal{L}_{2,2}^{1,1}$ and $6 \mathcal{L}_{3,1}^{1,1}$ and the axially symmetric configurations of two types, $\mathcal{A}_{32}$ and $\mathcal{A}_{61}$, respectively. As $g=0$, the link $6 \mathcal{L}_{2,2}^{1,1}$ represent the global minimum; it has a lower energy than other configurations in that sector for all values of $g$. An increase of the coupling constant and related magnetic interaction deforms this initial configuration into an axially symmetric soliton $6 \mathcal{A}_{32}$; as $g=1$, it has an energy about $16 \%$ lower than another axially symmetric Hopfion $6 \mathcal{A}_{61}$.

The trefoil knot $7 \mathcal{K}_{3,2}$ is the only minimizer in the sector of degree seven as $g=0$. However, as the gauge coupling grows, the magnetic fluxes associated with the loops $\mathcal{C}_{-}=$ $\vec{\phi}^{-1}(0,0,-1)$ tend to merge because of the attraction between them. At $g=1$, the knot becomes deformed into a configuration whose position curve represents two contacting loops, which are not interlinked; see Fig. 1. This structure is similar to the corresponding solution in the sector $Q=5$.

At degree $Q=8$, there are three energy minima, which represent a link $8 \mathcal{L}_{3,3}^{1,1}$, a knot $8 \mathcal{K}_{3,2}$, and axially symmetric Hopfions $8 \mathcal{A}_{42}$, respectively. As $g=0$, the link $\mathcal{L}_{3,3}^{1,1}$ has an energy a little less than the knot $8 \mathcal{K}_{3,2}$. However, as $g$ increases, the axial symmetry is recovered and the $8 \mathcal{A}_{4,2}$ becomes a global minimizer in that sector as $g=1$. This
Hopfion is composed of two $4 \mathcal{A}_{41}$ Hopfions stacked one above the other with the orientation in the maximally attractive channel. Another axially symmetric configuration $8 \mathcal{A}_{81}$ in the strong coupling limit has a bit higher energy. Again, we observe that the structure of the magnetic fluxes is completely determined by the preimages of $\mathcal{C}_{-}=$ $\vec{\phi}^{-1}(0,0,-1)$ and $\mathcal{C}_{+}=\vec{\phi}^{-1}(0,0,1)$; see Fig. 1 . As $g=1$, we find that the $8 \mathcal{K}_{3,2}$ Hopfion, similarly to that of $Q=7$ and $Q=5$, deforms into configurations with two contacting loops; see Fig. 1.

\section{B. Gauged Hopfions at $\vec{\phi}_{\infty}=(1,0,0)$}

Unlike the gauged planar baby-Skyrme model [27-29], in the three-dimensional Faddeev-Skyrme-Maxwell model (1), the $U(1)$ gauging prescription (3) is not necessarily correlated with the vacuum boundary condition imposed on the scalar field. The topological restriction on the scalar field is that on the spatial boundary $\vec{\phi}$ must approach the same vacuum value regardless of direction, and then $\mathbb{R}^{3} \mapsto S^{3}$.

On the other hand, the condition of finiteness of the energy of the system for any choice of the vacuum requires the same restrictions on the spatial infinity:

$$
D_{\mu} \vec{\phi}=0, \quad F_{\mu \nu}=0 .
$$

Choosing an appropriate gauge, we can just impose $\vec{\phi}_{\infty}=$ const.

In our consideration above, we suppose that the vector $\vec{n}=(0,0,1)$ and the vacuum $\vec{\phi}_{\infty}$ are parallel; let us consider another possibility by imposing the boundary condition [44] $\vec{\phi}_{\infty}=(1,0,0)$ with the gauging prescription (3). Evidently, as $g=0$, the choice of the vacuum does not affect the structure of the Hopfion solutions in the model (1) without a potential, for any particular choice of the $\vec{\phi}_{\infty}$, and the topological properties of the solitons are defined by 
the Hopf charge (2). However, as the gauge coupling increases, the difference between the directions of $\vec{\phi}_{\infty}$ and $\vec{n}$ leads to significant deformations of the configurations.

The results of energy minimization simulations are summarized in Fig. 2. We confirm that the structure of the magnetic field of the gauged Hopfion is always determined by the collection of loops $\mathcal{C}_{ \pm}=\phi^{-1}(0,0, \pm 1)$, for any value of the parameter $\beta$.

We observe that, as the parameter $\beta$ is increasing, the energy of the $Q=1$ Hopfion is decreasing; however, the static energy of the configurations of higher degrees is increasing. Rotation of the vacuum with respect to the direction of the vector $\vec{n}$ effectively deforms the soliton; as $g=1$ and $\vec{\phi}_{\infty}=(1,0,0)$, the $Q=1$ Hopfion is no longer axially symmetric, and the magnetic field represents two fluxes, which are linked once. Although the position curve of this Hopfion remains a single loop, the energy density distribution of the configuration at $g \sim 1$ looks more like the link; see Fig. 2.

Slightly deformed at $g=1$, unknot $2 \mathcal{A}_{21}$ is the minimal energy configuration in the sector $Q=2$; see Figs. 2 and 4 . Interestingly, as the gauge coupling increases, the position curve of the higher-energy solution $2 \tilde{\mathcal{A}}_{12}$ is split into two contiguous loops; see Fig. 2, third row. However, unlike the position curve of a link, there is no interlinking of two loops. Thus, this configuration can be labeled as $\mathcal{L}_{1,1}^{0,0}$. The magnetic fluxes, which correspond to the curve $\mathcal{C}_{+}=\phi^{-1}(0,0,1)$, are propagating in the same direction.

More generally, the splitting loops are touching each other without interlinking at the point where magnetic fluxes are parallel. Our calculations show that it may happen at the center of the Hopfion, like for $2 \mathcal{A}_{2,1}, 3 \mathcal{A}_{3,1}, 4 \mathcal{A}_{2,2}, 4 \mathcal{A}_{4,1}$, $6 \mathcal{A}_{6,1}$, and $8 \mathcal{A}_{4,2}$ configurations; see Fig. 2 . The point of contact can also be not at the center of the configuration; it happens for $2 \mathcal{A}_{1,2}, 5 \mathcal{L}_{1,2}$, and $6 \mathcal{A}_{2,2}$ Hopfions.

Another possibility is that the curves of $\mathcal{C}_{ \pm}=$ $\phi^{-1}(0,0, \pm 1)$ lie on top of each other; we observed this type of behavior for $3 \mathcal{A}_{3,1}, 4 \mathcal{A}_{2,2}, 4 \mathcal{A}_{4,1}$, and $5 \mathcal{L}_{1,2}$ Hopfions.

Considering the axially symmetric gauged Hopfions $\mathcal{A}_{i, j}$, we found that either they may form symmetric configurations with a single loop $\mathcal{C}_{-}=\phi^{-1}(0,0,-1)$, like $2 \mathcal{A}_{2,1}, 4 \mathcal{A}_{4,1}, 6 \mathcal{A}_{6,1}$, etc., or the blobs may appear on the loops, like $5 \mathcal{A}_{5,1}, 7 \mathcal{A}_{7,1}$, and $8 \mathcal{A}_{8,1}$ configurations; see Fig. 2. Notably, the $6 \mathcal{A}_{6,1}$ and $8 \mathcal{A}_{4,2}$ Hopfions possess $D_{6}$ symmetry and $D_{4}$ symmetries, respectively.

\section{Dependence on gauge coupling for $Q=5$}

As a particular example of the parametric dependency of the gauged Hopfions on the coupling constant $g$, we considered solitons in the sector of degree $Q=5$, in the case of both the vacuum $\vec{\phi}_{\infty}=(0,0,1)$ and $\vec{\phi}_{\infty}=(1,0,0)$.

In Fig. 4, we have plotted the graphs of total energy of the gauged $5 \mathcal{A}_{5,1}$ and $5 \mathcal{L}_{1,2}^{1,1}$ Hopfions, defined by the functional (6), and the magnetic energy as a function of the gauge coupling $g$. As $g=0$, both choices of the vacuum are equivalent, and the energy of the link $5 \mathcal{L}_{1,2}^{1,1}$ is lower than the axially symmetric Hopfion $5 \mathcal{A}_{5,1}$. However, as the gauge coupling increases from zero, the latter configuration becomes a global minimum in the vacuum $\vec{\phi}_{\infty}=(0,0,1)$, while the link $5 \mathcal{L}_{1,2}^{1,1}$ still remains the minimal energy solution in the vacuum $\vec{\phi}_{\infty}=(1,0,0)$, as seen in the left plot in Fig. 4.

A general observation is that, as the gauge coupling increases, the energy of the gauged Hopfion monotonically decreases. On the other hand, the magnetic energy initially increases from zero; it attains its maximum at $g \simeq 0.7$. Further increase of the coupling leads to a decrease of the magnetic energy, as shown in the right plot in Fig. 4. As expected, the size of the Hopfions decreases as the coupling $g$ increases.

In Figs. 5 and 6, we display the pattern of evolution of the initial $5 \mathcal{A}_{5,1}$ and $5 \mathcal{L}_{1,2}^{1,1}$ configurations, as the gauge coupling $g$ is growing from zero. In the model (1) with the usual choice of the vacuum $\vec{\phi}_{\infty}=(0,0,1)$, coupling to the

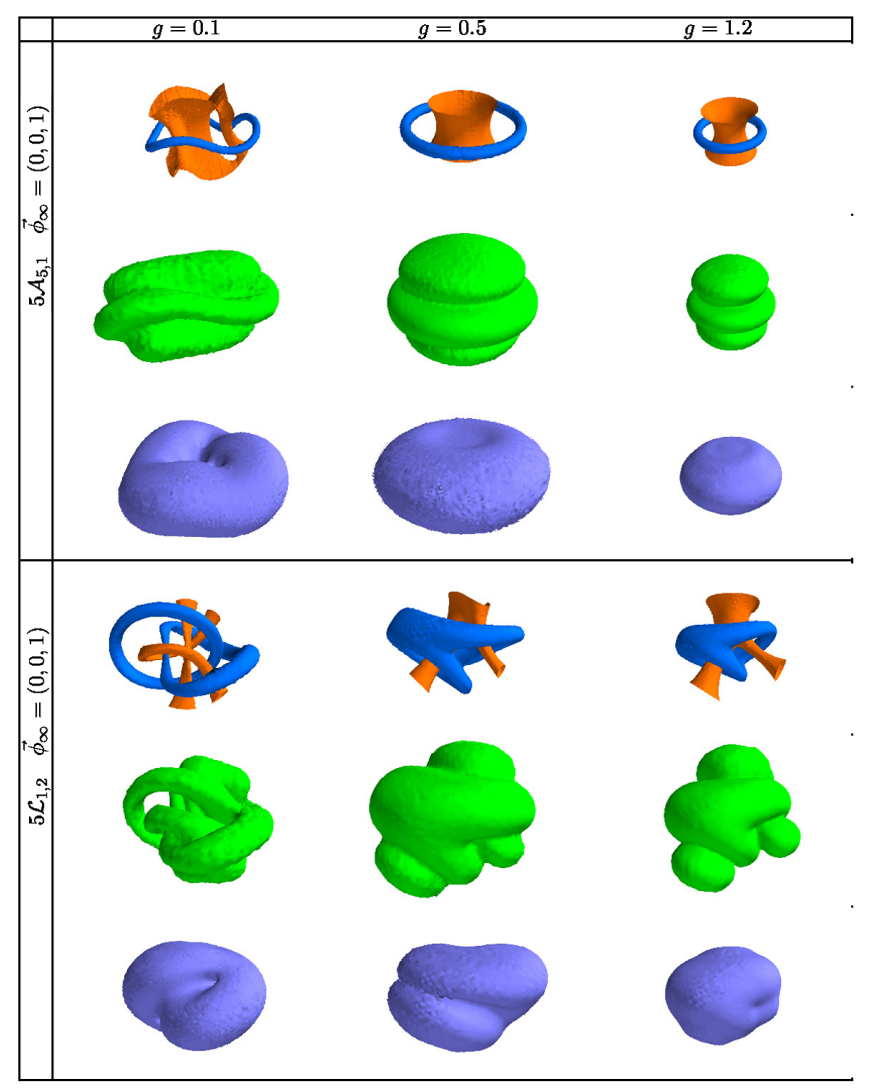

FIG. 5. Isosurfaces of the field components $\phi_{3}= \pm 0.9$ (first row), $|B|=1$ isosurfaces of the magnetic field (second row), and $E=2$ isosurfaces of the energy density (third row) of the $Q=5$ Hopfions in the model (6) with the vacuum $\vec{\phi}_{\infty}=(0,0,1)$ for $g=0.1, g=0.5$, and $g=1.2$. 


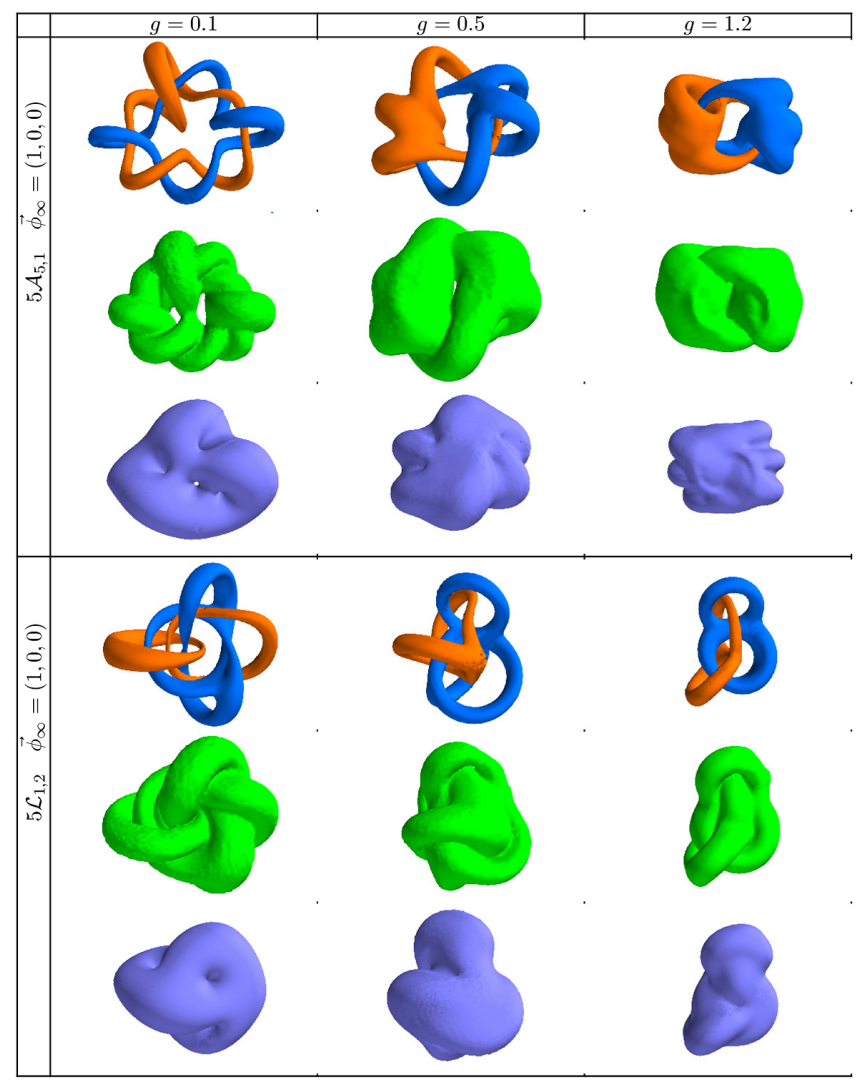

FIG. 6. Isosurfaces of the field components $\phi_{3}= \pm 0.9$ (first row), $|B|=1$ isosurfaces of the magnetic field (second row), and $E=2$ isosurfaces of the energy density (third row) of the $Q=5$ Hopfions in the model (6) with the vacuum $\vec{\phi}_{\infty}=(1,0,0)$ for $g=0.1, g=0.5$, and $g=1.2$.

magnetic field, directed along the vector $\vec{n} \| \vec{\phi}_{\infty}$, recovers the axial symmetry of the $5 \mathcal{A}_{5,1}$ Hopfion, which is violated as $g \lesssim 0.25$. Within that range of values of $g$, the bent axial solution $5 \tilde{\mathcal{A}}_{5,1}$ is a local energy minimum, and the minimal energy configuration in this sector remains the link $5 \mathcal{L}_{1,2}^{1,1}$. However, as $g \gtrsim 0.25$, the lowest-energy solution is the axially symmetric Hopfion $5 \mathcal{A}_{5,1}$. The magnetic fluxes of this configuration are directed through the center of the Hopfion and around the symmetry axis, as shown in Fig. 5, upper panel. In the strong coupling limit, the link $5 \mathcal{L}_{1,2}^{1,1}$ becomes strongly deformed; see Fig. 5, bottom panel. Magnetic attraction between the fluxes, associated with the position curve of the soliton, deforms the curve itself; for sufficiently large values of $g$, it is shaped as two contacting loops without interlinking. As we have seen above, the magnetic fluxes and the energy density distribution follow the curves $\mathcal{C}_{ \pm}=\phi^{-1}(0,0, \pm 1)$.

The pattern of the evolution of the $Q=5$ Hopfions in the (1) with the vacuum $\vec{\phi}_{\infty}=(1,0,0)$, following the increase of $g$, is somewhat different from what is outlined above. The position curve of the $Q=5$ axially symmetric configuration $\mathcal{A}_{5,1}$ gradually becomes deformed into a loop with internal twisting; see Fig. 6, upper panel. The link $5 \mathcal{L}_{1,2}^{1,1}$ has a lower energy as $g \gtrsim 0.4$, the position curve of this configuration, is deformed into two twisted unlinked adjoining rings.

\section{CONCLUSIONS}

The objective of this work is to investigate properties of soliton solutions of the Faddeev-Skyrme-Maxwell model. We have considered the Hopfion solutions with topological charges up to $Q=8$, coupled to the magnetic field. We found that, as the gauge coupling increased, the backreaction of the magnetic field may significantly affect the structure of the solutions; however, the results depend both on the type of the vacuum boundary condition and on the strength of the gauge coupling. We found that the magnetic fluxes of gauged Hopfions follow the directions provided by preimages of the vectors $\vec{\phi}=(0,0, \pm 1)$. In the strong coupling limit, the magnetic field of the gauged Hopfion exhibits behavior similar to the field of the vortex solution of the Abelian Higgs model.

The work here should be taken further by considering the electrically charged configurations; another interesting direction is to investigate the soliton solutions of the $S O$ (3) gauged Faddeev-Skyrme model. It might be also interesting to consider gauged Hopfions in frustrated magnets, which combine nearest-neighbor ferromagnetic and higher-neighbor antiferromagnetic interactions [45]. We hope we can address these issues in our future work.

\section{ACKNOWLEDGMENTS}

Y. S. gratefully acknowledges support from the Ministry of Education and Science of Russian Federation, Project No. 3.1386.2017. The parallel computations were performed on the cluster HIBRILIT at LIT, JINR, Dubna. 
[1] A. A. Bogolubskaya and I. L. Bogolubsky, Phys. Lett. A 136, 485 (1989); Lett. Math. Phys. 19, 171 (1990).

[2] R. A. Leese, M. Peyrard, and W. J. Zakrzewski, Nonlinearity 3, 773 (1990).

[3] T. H. R. Skyrme, Proc. R. Soc. A 260, 127 (1961).

[4] C. Adam, J. Sanchez-Guillen, and A. Wereszczynski, Phys. Lett. B 691, 105 (2010).

[5] C. Adam, J. Sanchez-Guillen, and A. Wereszczynski, Phys. Rev. D 82, 085015 (2010).

[6] L. D. Faddeev, Quantization of Solitons, Preprint-75-0570 (IAS, Princeton, 1975).

[7] L. D. Faddeev and A. J. Niemi, Nature (London) 387, 58 (1997).

[8] G. H. Derrick, J. Math. Phys. (N.Y.) 5, 1252 (1964).

[9] D. Foster, Phys. Rev. D 83, 085026 (2011).

[10] P. Sutcliffe, J. High Energy Phys. 04 (2011) 045.

[11] L. A. Ferreira and Y. Shnir, Phys. Lett. B 772, 621 (2017).

[12] L. A. Ferreira, J. High Energy Phys. 07 (2017) 039.

[13] N. Manton and P. Sutcliffe, Topological Solitons (Cambridge University Press, Cambridge, England, 2004).

[14] Y. M. Shnir, Topological and Non-Topological Solitons in Scalar Field Theories (Cambridge University Press, Cambridge, England, 2018).

[15] M. Kobayashi and M. Nitta, Phys. Lett. B 728, 314 (2014).

[16] R. A. Battye and P. M. Sutcliffe, Nucl. Phys. B705, 384 (2005).

[17] R. A. Battye, N. S. Manton, P. Sutcliffe, and S. W. Wood, Phys. Rev. C 80, 034323 (2009).

[18] P. Salmi and P. Sutcliffe, J. Phys. A 48, 035401 (2015).

[19] S. B. Gudnason and M. Nitta, Phys. Rev. D 94, 065018 (2016).

[20] S. B. Gudnason, B. Zhang, and N. Ma, Phys. Rev. D 94, 125004 (2016).

[21] J. Jaykka and M. Speight, Phys. Rev. D 82, 125030 (2010).

[22] M. Kobayashi and M. Nitta, J. Low Temp. Phys. 175, 208 (2014).

[23] M. Kobayashi and M. Nitta, Phys. Rev. D 87, 125013 (2013).

[24] S. B. Gudnason and M. Nitta, Phys. Rev. D 91, 085040 (2015).
[25] Á. Lukács, J. Math. Phys. (N.Y.) 57, 072903 (2016).

[26] A. Samoilenka and Y. Shnir, J. High Energy Phys. 09 (2017) 029.

[27] J. Gladikowski, B. M. A. G. Piette, and B. J. Schroers, Phys. Rev. D 53, 844 (1996).

[28] A. Samoilenka and Y. Shnir, Phys. Rev. D 93, 065018 (2016).

[29] C. Adam and A. Wereszczynski, Phys. Rev. D 95, 116006 (2017).

[30] J. Gladikowski and M. Hellmund, Phys. Rev. D 56, 5194 (1997).

[31] R. Battye and P. Sutcliffe, Phys. Rev. Lett. 81, 4798 (1998).

[32] P. Sutcliffe, Proc. R. Soc. A 463, 3001 (2007).

[33] Y. Shnir and G. Zhilin, Phys. Rev. D 89, 105010 (2014).

[34] B. J. Schroers, Phys. Lett. B 356, 291 (1995).

[35] Y. Shnir and D. H. Tchrakian, J. Phys. A 43, 025401 (2010).

[36] A. Samoilenka and Y. Shnir, Phys. Rev. D 97, 045004 (2018).

[37] More precisely, the direction of the second flux is given by the vector $\vec{n}$, which we introduced in the definition of the covariant derivative (3).

[38] A. Samoilenka and Y. Shnir, Phys. Rev. D 95, 045002 (2017).

[39] D. Harland, J. Jäkkä, Y. Shnir, and M. Speight, J. Phys. A 46, 225402 (2013).

[40] R. A. Battye and M. Haberichter, Phys. Rev. D 87, 105003 (2013).

[41] J. Hietarinta and P. Salo, Phys. Lett. B 451, 60 (1999).

[42] R. S. Ward, Phys. Lett. B 473, 291 (2000).

[43] G. E. Marsh, Force-Free Magnetic Fields: Solutions, Topology and Applications (World Scientific, Singapore, 1996).

[44] More generally, we can consider a continuous family of vacua

$$
\vec{\phi}_{\infty}=(\sin \beta, 0, \cos \beta)
$$

where parameter $\beta \in[0,2 \pi]$.

[45] P. Sutcliffe, Phys. Rev. Lett. 118, 247203 (2017). 